\title{
UNIVERSO REPRESENTACIONAL DEL ARTE RUPESTRE DEL SITIO LOS MELLIZOS (PROVINCIA DEL CHOAPA): CONVENCIONES VISUALES Y RELACIONES CULTURALES
}

\section{REPRESENTATIONAL UNIVERSE OF LOS MELLIZOS ROCK ART SITE ICHOAPA PROVINCE): VISUAL CONVENTIONS AND CULTURAL RELATIONS}

\section{Paola González Carvajal *}

\begin{abstract}
Este trabajo examina nuevos caminos metodológicos para el estudio de la iconografía del arte rupestre del sitio Los Mellizos. En particular, se emplean los principios geométricos de la simetría en la descripción y la comprensión de este arte visual. Luego se clasifican sus formas y estructuras subyacentes. Al mismo tiempo, la estructura del diseño ha demostrado ser un atributo sensible a problemas relacionados con la identidad de grupo y los procesos de intercambio e interacción. Para abordar este último aspecto, un segundo esfuerzo fue realizado al comparar los patrones decorativos de Los Mellizos con patrones decorativos de la decoración cerámica de culturas prehispánicas presentes en la región. Como resultado de este ejercicio identificamos una fuerte presencia de iconografía Inka o Diaguita-Inka, así como otras influencias culturales.
\end{abstract}

Palabras clave: universo representacional, arte rupestre, Cultura Diaguita, análisis de simetría, aproximación metodológica

This paper examines new methodological approaches to the systematic study of Los Mellizos rock art iconography. In particular, we use the geometrical principles of symmetry to describe and understand this visual art, then classify the forms and underlying structures. At the same time, the structure of the design has been shown to be sensitive to issues of group identity, exchange and interaction. Therefore, a secondary effort compares Los Mellizos decorative patterns with ceramic decorative patterns of the pre-Hispanic cultures present in the region. As a result of this exercise, we identified the strong presence of Inka and Diaguita-Inka visual patterns and other cultural influences.

Key words: representational universe, rock art, Diaguita Culture, symmetry analysis, methodological approach
A continuación se presentan los resultados del estudio del arte rupestre (petroglifos) que se encuentra en el sitio Los Mellizos localizado en el curso superior del valle de Illapel (provincia del Choapa, Región de Coquimbo). ${ }^{1}$ El valle de Illapel forma parte del Norte Semiárido chileno y se localiza en la frontera sur de este espacio geográfico. Por su extensión longitudinal y amplitud ha servido de lugar de asentamiento a numerosas poblaciones prehispánicas, especialmente a partir del Período Agroalfarero (100 DC). Este valle cuenta con numerosos pasos cordilleranos que lo comunican con valles allende los Andes (provincia de San Juan, Argentina). Estas vías de comunicación fueron utilizadas por las poblaciones precolombinas y continúan siendo transitadas por arrieros actuales, siguiendo el sistema de veranadas. En las últimas décadas, el arte rupestre de la provincia del Choapa ha recibido una creciente atención (Cabello 2001; Jackson et al. 2001; Artigas \& Cabello 2004).

En particular, el arte rupestre del sitio Los Mellizos ha despertado el interés de diversos investigadores (Castillo 1991; Toro 1996; Ballereu \& Niemeyer 1998; Troncoso 2001), cada uno de los cuales enfatizó diferentes aspectos en la comprensión de este sitio arqueológico. Destacamos especialmente el enfoque de Troncoso (2001: 15) quien, mediante una aproximación espacial e iconográfica, plantea que el sitio Los Mellizos juega un importante rol en el proceso de construcción social 
del espacio durante el Período Agroalfarero Temprano (PAT) en el valle de Illapel. El autor señala que este sitio arqueológico (Los Mellizos) presenta un "carácter heterotópico" (sensu Foucault 1986) en atención a su cultura material y ubicación espacial. ${ }^{2}$ Esta afirmación se basa en tres aspectos: a) ubicación del sitio en un área de frontera donde finaliza el valle y comienza la cordillera; b) presencia de gran cantidad de paneles de arte rupestre, siendo que en las restantes estaciones rupestres el número de paneles no excede la treintena, y c) complejidad iconográfica de los paneles, donde se registran "motivos extremadamente complejos en su diseño y elaboración” (Troncoso 2001: 15).

La presente aproximación busca aportar nuevas luces al conocimiento del sitio Los Mellizos mediante un análisis pormenorizado de sus expresiones iconográficas. Se propone la aplicación de una metodología que atiende a la caracterización de formas y lógica composicional (estructuración) de los motivos al interior del panel, mediante un enfoque teórico-metodológico que a continuación se detalla.

\section{METODOLOGÍA APLICADA AL ESTUDIO DEL UNIVERSO REPRESENTACIONAL DE LA ESTACIÓN RUPESTRE LOS MELLIZOS}

En primer término, es necesario precisar que nuestra aproximación se focaliza en las representaciones iconográficas presentes en los paneles de arte rupestre. No obstante, el arte rupestre -dado su carácter material, espacial y visual- debe ser entendido como un objeto de estudio multifacético, por lo cual no solamente la iconografía aporta información relevante para su comprensión. En efecto, existe un conjunto de entradas de información que son también relevantes para su estudio. Troncoso y colaboradores (2008) realizan una serie de proposiciones metodológicas para la comprensión del arte rupestre a partir de trabajos anteriores del mismo autor (Troncoso 2005) y de los aportes de Tilley (1991) y Santos (2005). Así, en este trabajo se plantea la distinción entre atributos intrínsecos y extrínsecos para el análisis del arte rupestre. Debe entenderse por atributos intrínsecos aquellos inherentes a la iconografía propiamente tal (motivos y estructuración en el panel), así como a las técnicas de producción, y a las relaciones espaciales "establecidas entre los diseños, entre los bloques y entre los sitios de arte rupestre" (Troncoso et al. 2008: 14). De acuerdo al trabajo referido, los atributos intrínsecos son de la mayor relevancia para entender la variabilidad del arte rupestre y la discriminación de conjuntos diferentes (o estilos). En este trabajo se abordará en detalle un área de los atributos intrínsecos, específicamente la iconografía de los paneles, desde una perspectiva metodológica que enfatiza la forma de estas representaciones y su configuración en el espacio del panel. Este análisis no aborda las relaciones espaciales entre bloques ni la técnica de producción.

Los atributos extrínsecos, en tanto, dependen de las dinámicas sociales de los distintos grupos humanos y no serían tan gravitantes en la asignación cronocultural del arte rupestre, ni en su sistematización. Estos atributos incluyen, por ejemplo, la asociación con depósitos arqueológicos o asentamientos habitacionales y se refieren a la contigüidad espacial. Los atributos extrínsecos no bastan por sí mismos para establecer la vinculación cultural de un determinado arte rupestre, es necesario considerar previamente los atributos intrínsecos.

La propuesta metodológica de Troncoso y colaboradores (2008) para el análisis del arte rupestre, con la cual concordamos, plantea la necesidad de usar distintas líneas argumentativas para enriquecer la interpretación de este objeto de estudio. Es decir, debemos considerar la exploración de "diferentes aspectos del arte rupestre con el fin de construir cables argumentativos, donde los atributos intrínsecos siempre serán de una valoración mayor" (Troncoso et al. 2008: 15).

A partir de este contexto, a continuación ahondaremos en una aproximación metodológica que aborda de lleno uno de los componentes de los atributos intrínsecos que consideramos muy relevante en la comprensión del arte rupestre, nos referimos a la iconografía. A este respecto, la determinación de las unidades mínimas que serán estructuradas simétricamente en el campo del diseño (panel de arte rupestre) puede contribuir grandemente a establecer la variabilidad iconográfica de dicho arte y las reglas que gobiernan su composición. El enfoque metodológico propuesto en este trabajo presta atención a la organización de los motivos dentro del espacio del panel, ampliando el enfoque centrado solo en las unidades de diseño en sí mismas. Esta aproximación complementa un conjunto de nuevos enfoques teóricos y metodológicos para el estudio del arte rupestre en Chile (Gallardo et al. 2006; Gallardo 2009). También aporta a la asignación cultural del arte rupestre al hacer posible la comparación sistemática de las estructuras decorativas presentes en este con patrones simétricos presentes en otros soportes arqueológicos de datación conocida. 


\section{APORTE DEL ANÁLISIS DE SIMETRÍA EN LA CARACTERIZACION DE ICONOGRAFÍA DEL ARTE RUPESTRE}

Una primera idea que aporta a la presente discusión y que guía nuestra propuesta metodológica se refiere a que el concepto de simetría está siempre relacionado con la idea de categorización, con la clasificación de regularidades observadas en el mundo sensible, lo que, en último término, se relaciona con la noción de límites. No obstante, de acuerdo a Wade (2006) en sí misma la simetría es ilimitada; no existe ningún lugar que no sea penetrado por estos principios. Además, estos se caracterizan por la quietud o detención, pero también se relacionan con las ideas de transformación o movimiento. También es importante tener presente que cualquier concepto de simetría está siempre vinculado con el de asimetría, así como con la oposición entre orden y desorden. Estas afirmaciones aportan a la justificación de nuestra primera opción metodológica que busca la caracterización del universo representacional del arte rupestre del sitio Los Mellizos. Un primer paso fue separar este universo representacional entre diseños simétricos y no simétricos. Para dar cuenta de la totalidad de los motivos que componen este arte rupestre, distinguimos entre formas geométricas y no geométricas. Luego, establecimos dos categorías dicotómicas que están por encima de las recién reseñadas y que se refieren a la naturaleza figurativa y no figurativa de los motivos que integran este universo representacional.

Cuando reflexionamos acerca del rol de la simetría en el arte, una primera constatación se refiere a su universalidad y su vinculación con el concepto de estilo. De acuerdo a Wade (2006: 46) donde sea que exista simetría en el arte, ella estará íntimamente relacionada con las particularidades de un estilo, dado que la simetría implica siempre un principio ordenador u organizador. El autor plantea que los seres humanos somos criaturas conscientes de la simetría, somos buscadores de patrones (pattern-seekers) por naturaleza. Así, los principios de simetría no estarán nunca enteramente ausentes del arte, pese a que existen grandes variaciones culturales en el rol de la simetría en las expresiones artísticas. En algunas culturas esta juega un rol pequeño, mientras que en otras existe una obsesiva exploración de sus posibilidades. Comparar esta fascinación (o ausencia de ella) en un amplio rango de sociedades es un ejercicio interesante. De hecho, aunque la mayor parte de las culturas, en distintos períodos y lugares del mundo, han usado patrones simétricos como parte de su repertorio decorativo, algunas de ellas parecen haber estado especialmente dedicadas a desarrollar los patrones simétricos como una forma de expresión artística (por ejemplo, el arte islámico, la iconografía celta). Wade (2006: 48) destaca que el empleo de patrones regulares involucra una medición del espacio a ser decorado. A partir de ello el artista, consciente o inconscientemente, se ve confinado a ajustarse a las reglas que gobiernan el tipo específico de simetría que está empleando. Estas limitaciones, sin embargo, siempre dejan abierta la posibilidad de incorporar cambios, generando variabilidad al interior del patrón. De este modo, la exploración (muchas veces inconsciente pero también sistemática) de los distintos tipos de simetría pareciera finalmente desdibujar la distinción existente entre la actividad artística de creación de patrones y la reflexión científica, cuyo objeto es la detección de nuevos patrones.

Profundizando un poco más en el análisis cultural de la simetría, destacamos que las estructuras simétricas nos comunican información porque son parte de un sistema mayor; lo integran y, a la vez, pueden llegar a ser una representación de él. En este sentido, el arte es redundante, comunica y repite información expresada en otras áreas de la cultura. Según Washburn y Crowe (1988: 15),

\footnotetext{
la simetría es una propiedad culturalmente significativa por dos razones. Por un lado señala elecciones culturales destinadas a seleccionar determinados principios simétricos para estructurar los diseños contribuyendo al proceso de autoidentificación de una cultura dada y, por otro lado, permite un acercamiento sistemático a los procesos de intercambio e interacción estilísticos entre culturas diferentes.
}

\section{PROCEDIMIENTO DE ANÁLISIS DE SIMETRIA}

De acuerdo a Washburn $(1977,1983)$, el primer paso en el análisis de la simetría de un diseño es la definición de sus unidades fundamentales. Se entiende por tales a las partes asimétricas básicas que logran identidad consigo mismas, siguiendo uno de los principios que a continuación se reseñan.

Traslación: implica el movimiento simple de una parte fundamental a lo largo de la línea eje.

Rotación: requiere que la parte fundamental sea movida alrededor del punto eje. Las partes fundamentales pueden cambiar de orientación en cualquier punto dentro del arco de 360 grados.

Reflexión: requiere que la parte fundamental sea reflejada a través de la línea eje en una relación de imagen de espejo.

Reflexión desplazada: combina las nociones de reflexión tipo espejo, a través de la línea eje y la traslación. Este principio produce una figura que recuerda los 
movimientos alternados, derecha-izquierda, involucrados en la movilidad humana.

Los cuatro conceptos vistos son usados para generar todos los patrones simétricos. Además, existirían tres principales categorías de diseño para un patrón de diseño plano.

Finito: son figuras únicas generadas alrededor de un único punto eje de línea media.

Unidimensional: son generadas a lo largo de un único eje de línea media.

Bidimensional: son generadas a lo largo de ambos ejes, horizontal (x) y vertical (y).

Una vez establecidas estas unidades y configuraciones estructurales que articulan el universo visual del panel, el paso siguiente es asociar esta información a otros atributos intrínsecos (Troncoso 2005), a fin de establecer agrupaciones o conjuntos diferenciales dentro del universo rupestre, que den cuenta de lógicas de producción específicas (conjuntos estilísticos).

\section{RESULTADOS DEL ANÁLISIȘ DE FORMAS Y SIMETRIAA DE LA ESTACIÓN RUPESTRE LOS MELLIZOS}

En el marco del Proyecto Fondecyt 1080360, se definieron los patrones decorativos que gobiernan el universo representacional del arte rupestre de Los
Mellizos, presentes en 176 bloques de petroglifos. En términos generales, buscamos establecer la lógica interna que subyace a este arte visual, desde un punto de vista formal. Esta lógica se expresa en la elección de determinadas formas para la construcción de los diseños y, en el caso de aquellos diseños de naturaleza simétrica, se refleja también en la elección de determinadas estructuras de diseño que actúan sobre dichas formas, distribuyéndolas en el espacio del panel. De igual modo, una vez identificadas estas estructuras de diseño se procedió a compararlas con otros patrones decorativos, previamente establecidos, provenientes de la iconografía cerámica o rupestre (Gordillo 1990, 2009; Tarragó et al. 1997; Fiadone 2006; González 2008; Nastri 2008), de culturas prehispánicas que tuvieron presencia en el Norte Semiárido, con el objeto de proponer asignaciones culturales específicas para estos diseños rupestres. En definitiva, se analizaron 647 diseños y se establecieron sus patrones decorativos, considerando diseños figurativos (figuras antropomorfas, zoomorfas y artefactos) y diseños no figurativos (abstractos). En la muestra analizada, al interior de los diseños figurativos, se reconocieron dos grandes categorías: figurativos geométricos simétricos y figurativos geométricos no simétricos. En los diseños no figurativos se registraron las siguientes categorías: geométricos simétricos; geométricos no simétricos; no geométricos simétricos, y no geométricos no simétricos (fig. 1).
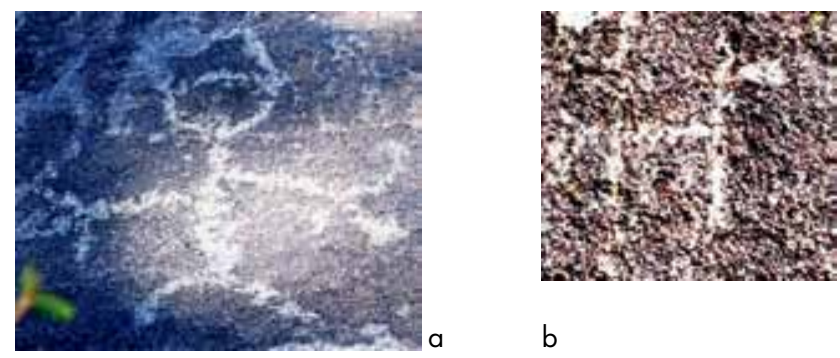

b
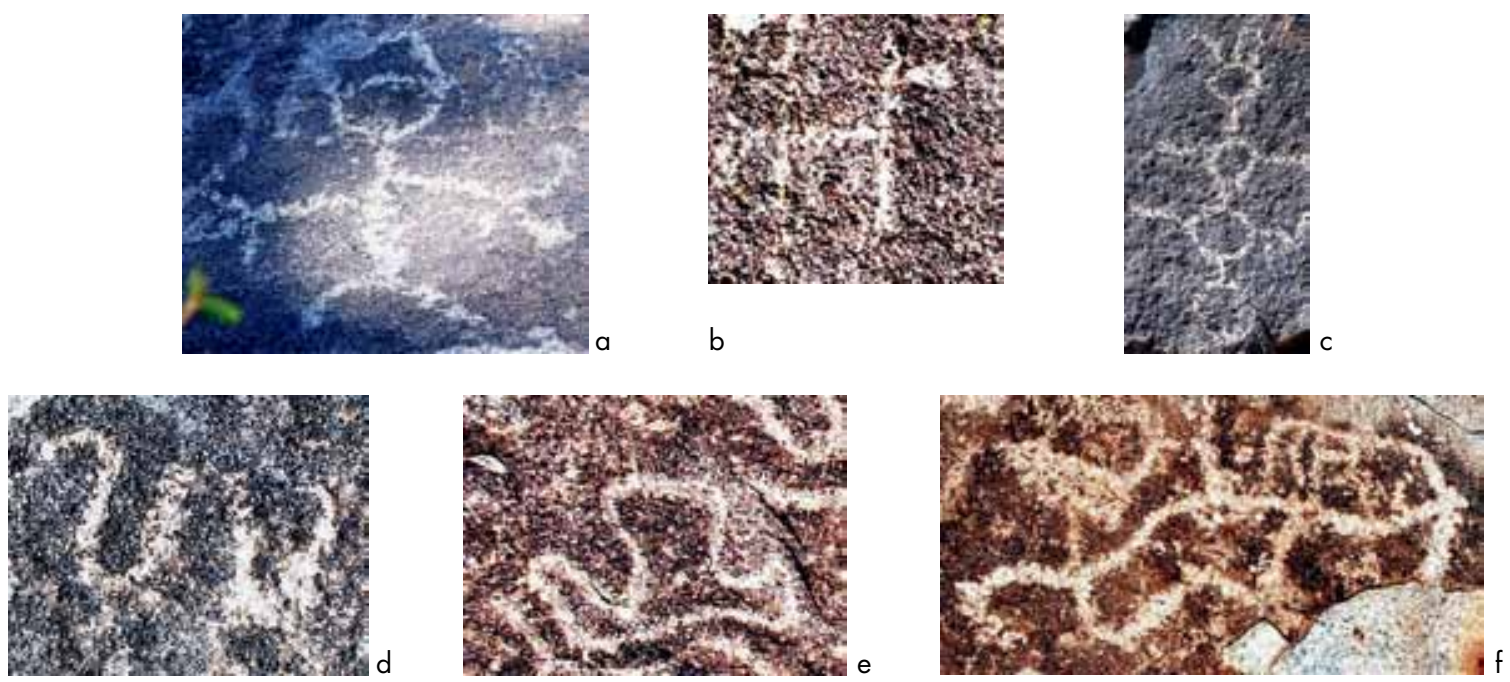

Figura 1. Ejemplos de categorías de diseños figurativos y no figurativos: a) Diseños figurativos geométricos simétricos: antropomorfo, soporte 43, panel A; b) Diseños figurativos geométricos no simétricos, soporte 29, panel B; c) Diseños no figurativos geométricos simétricos: círculos, curvas y rectas en reflexión vertical, soporte 37; d) Diseños no figurativos geométricos no simétricos: línea ondulada, soporte 72 ; e) Diseños no figurativos no geométricos simétricos, soporte 76, panel A; f) Diseños no figurativos no geométricos no simétricos, soporte 28. Figure 1. Examples of categories of figurative and non-figurative designs: a) Geometrical symmetrical figurative designs: human figure, support 43, panel A; b) Geometric asymmetrical figurative designs, support 29, panel B; c) Geometrical symmetrical non-figurative designs: circles, curves and straight lines in vertical reflection, support 37; d) Geometrical asymmetrical non-figurative designs: wavy line, support 72; e) Non-geometrical symmetrical non-figurative designs, support 76 , panel A; f) Non-geometrical asymmetrical non-figurative designs, support 28. 


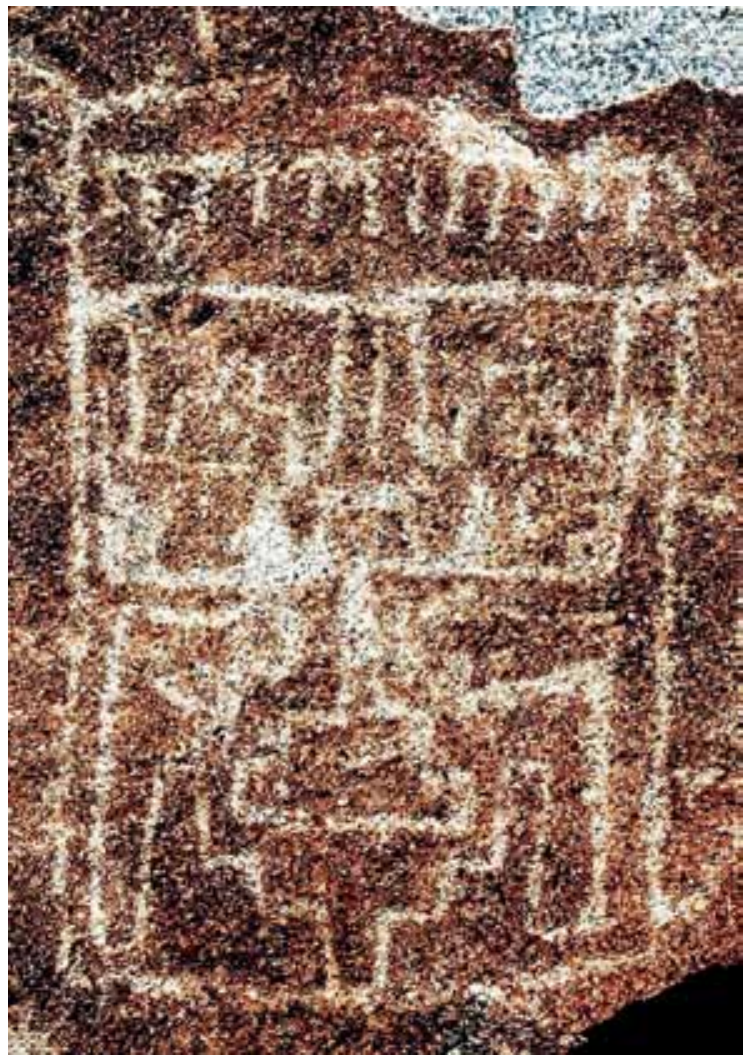

Figura 2. Diseños de filiación Diaguita en el arte rupestre del sitio Los Mellizos, soporte 41.

Figure 2. Designs showing Diaguita influence in the rock art of Los Mellizos, support 41

En cuanto a las filiaciones culturales identificadas, estas consideran diseños Diaguita (fig. 2); diseños Inka o Diaguita-Inka (fig. 3); diseños Aguada (figura 4a); diseños Ciénaga (figura 4b) y diseños santamarianos (figs. 4c y d).

Nuestra aproximación al estudio de la totalidad de la iconografía presente en esta estación rupestre reveló la existencia de un alto porcentaje de diseños de naturaleza no figurativa o abstracta, que alcanza a un $77,74 \%$ del total (503 diseños), en desmedro de los diseños figurativos. No obstante, en esta última categoría destacan importantes convenciones que dan cuenta de elecciones culturales que regulan las representaciones antropomorfas y zoomorfas.

\section{Diseños figurativos}

Se registraron un total de 144 diseños figurativos, de los cuales un 94,44\% (136 diseños) corresponde a diseños geométricos simétricos, caracterizados por un alto grado de simpleza y estilización. Aunque los atributos característicos de hombres y animales se reducen a su mínima expresión, no dejan de ser reconocidos como tales. Los ejecutores de este arte arribaron a convenciones esquemáticas y esenciales para representar seres antropomorfos y animales que, sin duda, desempeñaron un importante rol en su vida cotidiana.

Respecto a las figuras antropomorfas representadas de cuerpo entero (46 diseños), destacamos la existencia de ciertos patrones predominantes. Por una parte, un $100 \%$ de estas representaciones antropomorfas se construye según principio de reflexión vertical, esto le da al diseño una notoria rigidez, como opuesto a situaciones en que el cuerpo humano se representa en actividades dinámicas. También es interesante notar que dentro del universo de figuras antropomorfas regidas por reflexión vertical (un total de 13 variantes) dos de ellas alcancen una alta representación. Nos referimos a la categoría denominada "antropomorfo generado a partir de un círculo lleno, línea recta y apéndices rectos o curvos", y también a la categoría "antropomorfo generado a partir de un círculo vacío, línea recta y apéndices curvos o rectos", que en conjunto agrupan al $38,97 \%$ de los antropomorfos representados de cuerpo entero ( 46 diseños). Esto demuestra que, pese a la alta variabilidad, existe también un esfuerzo estandarizador con relación a determinados patrones de representación de la figura humana.

Es también en el ámbito de las representaciones antropomorfas donde es posible plantear algunas filiaciones culturales de quienes ejecutaron este arte rupestre. Nos referimos, por ejemplo, a las representaciones antropomorfas de origen santamariano, tres en total (ver figs. $4 \mathrm{C}$ y d). En ellas la figura humana se representa de frente, distinguiéndose un cuerpo rectangular con un estrechamiento en el sector medio, decorado interiormente mediante líneas horizontales paralelas (fig. 4c) o con la superficie raspada para generar un cambio de color (fig. 4d). Presentan también una cabeza con tocado de líneas oblicuas. Estos diseños son muy semejantes a ciertas figuras humanas propias de la iconografía santamariana en cerámica (ver Nastri 2008: 21, fig. 14) y arte rupestre (Tarragó et al. 1997: 231, fig. 6).

Asimismo, contamos con un diseño que evidencia una estrecha semejanza formal con las representaciones antropomorfas registradas en la cerámica diaguita-inka, nos referimos a la categoría denominada "antropomorfo generado por un cuadrado con rectángulos ajedrezados y apéndices rectos" (fig. 3a). De igual modo, destaca el conjunto de representaciones antropomorfas presentes en el soporte 78 , donde se observa una figura humana con un hacha en sus manos, aledaña a dos figuras antropomorfas notoriamente más pequeñas, pensamos que puede tratarse de una representación 

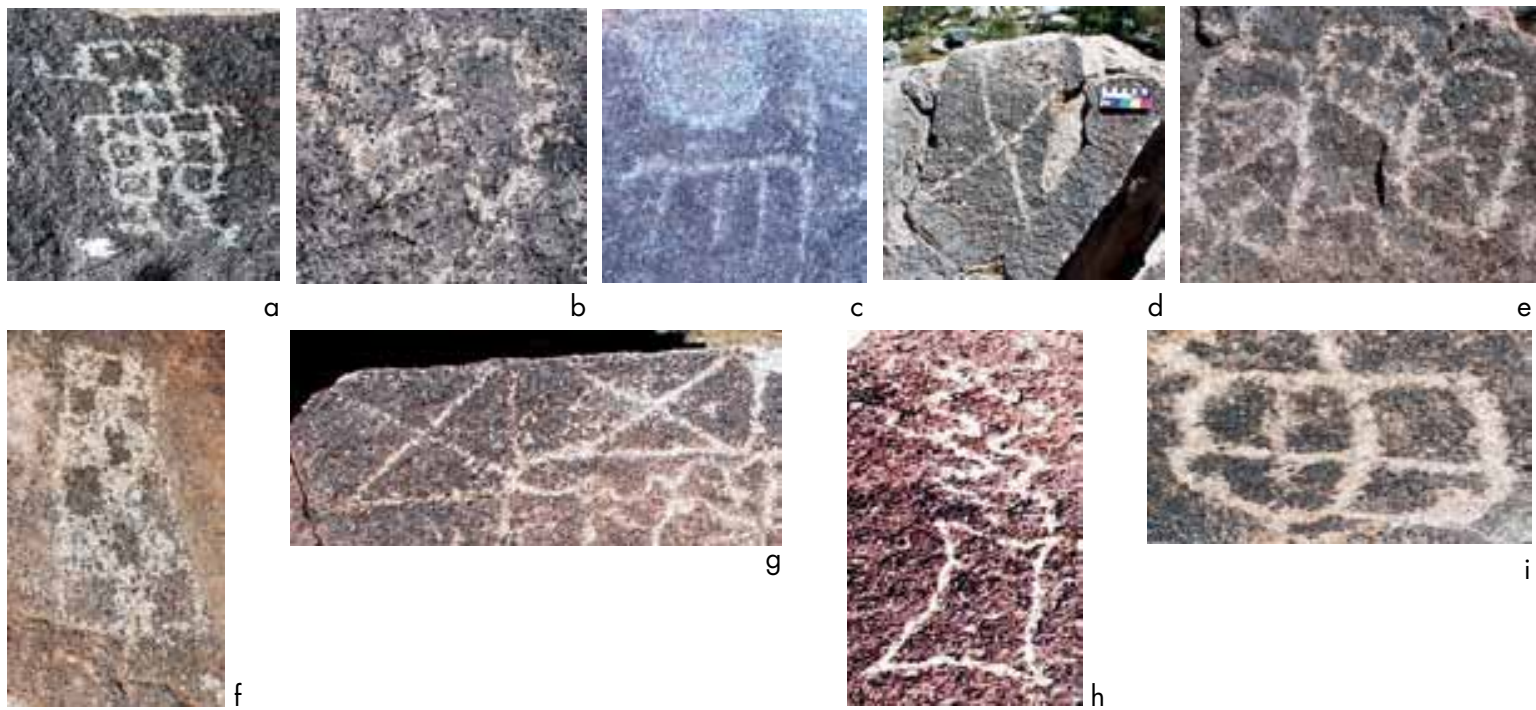

a

\begin{abstract}
b
\end{abstract}

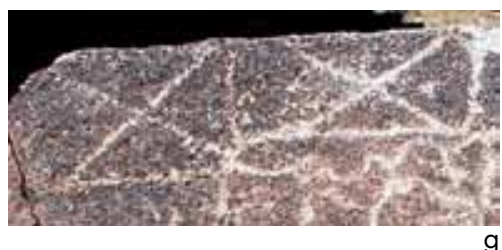

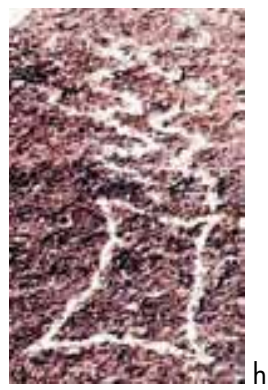

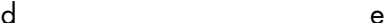

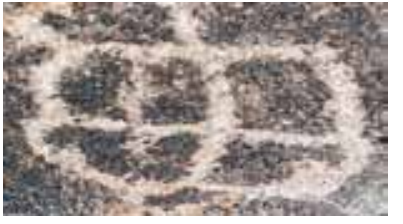

i
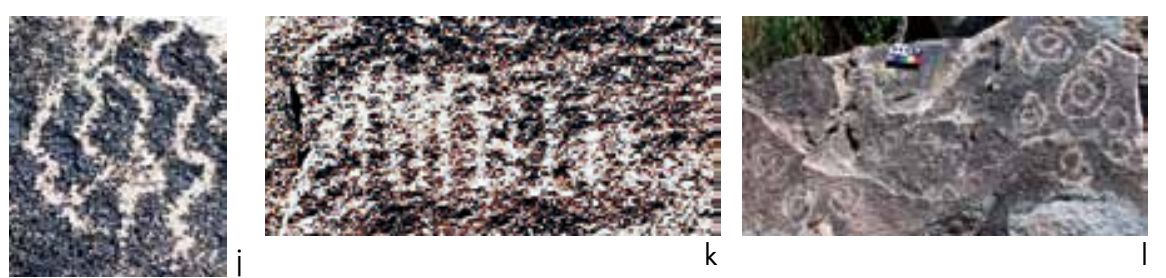

Figura 3. Algunos diseños de filiación cusqueña o Diaguita-Inka en el arte rupestre del sitio Los Mellizos: a) Antropomorfo con cuerpo ajedrezado, soporte 125; b) Tumi, soporte 120; c) Camélido estilizado, soporte 34; d) Clepsidra, soporte 91; e) Traslación rectángulos con cruz inscrita, soporte 124; f) Rectángulo ajedrezado, soporte 117; g) Traslación rectángulos con cruz inscrita, soporte 93; h) Rectángulo de paredes inflectadas, soporte 106; i) Cuadrado cruz inscrita, soporte 63; j) Traslación horizontal líneas onduladas, soporte 40; k) Greca inka, soporte 29; 1) Traslación horizontal y vertical de círculos concéntricos, soporte 97.

Figure 3. Some designs showing Inka or Diaguita-Inka influence in the rock art of Los Mellizos: a) Human figure with checkerboard body, support 125; b) Tumi, support 120; c) Stylized camelid, support 34; d) Hourglass, support 91; e) Duplicate rectangles with inscribed cross, support 124; f) Checkerboard rectangle, support 117; g) Duplicate rectangles with inscribed cross, support 93; b) Rectangle with concave walls, support 106; i) Square with cross inscribed, support 63; j) Horizontal wavy lines, support 40; $k$ ) Inka border, support 29; l) Horizontal and vertical placement of concentric circles, support 97.

de "El Sacrificador". En cuanto a la asignación cultural de estas representaciones, planteamos su vinculación con el Período Diaguita-Inka, dado que en el mismo panel se observa la representación de un tumi (fig. 6).

En cuanto a la categoría de representaciones antropomorfas, denominada "máscaras", varias de ellas aportan indicios acerca de su probable asignación cultural. Por ejemplo, las máscaras que evidencian el rasgo de "ceja continua", con ojos representados como círculos llenos, pueden ser asociadas a patrones decorativos cerámicos del PAT (figs. $5 \mathrm{a}$ y b), que recurren al empleo de modelados e incisos. Si bien este rasgo de ceja continua y ojos circulares no basta por sí mismo para asignarlo al PAT, sí lo serían otros rasgos presentes en su diseño. En efecto, Cabello (2001: 1364) describe máscaras semejantes dentro del Tipo I, caracterizadas por ser máscaras curvilíneas, de contorno circular, ojos y boca curvilíneos y con segmentación en la parte media del rostro. La autora asocia el Tipo I al PAT, postulado al cual adscribimos. También mencionaremos las máscaras de contorno rectangular con decoración interior de diseños escalonados y simetría en espejo vertical, asignable por sus unidades mínimas de diseño y estructura simétrica a los patrones decorativos de la Cultura Diaguita (fig. 2, Tabla 1).

Tabla 1. Diseños geométricos simétricos figurativos con influencia Diaguita o Diaguita-Inka: 4.

Table 1. Geometric symmetrical figurative designs with Diaguita or Diaguita-Inka influence: 4.

\begin{tabular}{|c|c|c|}
\hline Principio simétrico & Diseño & Total \\
\hline Reflexión vertical & Máscaras rectangulares con & \\
\hline Antropomorfo & $\begin{array}{c}\text { escalonados a modo de } \\
\text { ojos o boca }\end{array}$ & 4 \\
\hline Total & & 4 \\
\hline
\end{tabular}




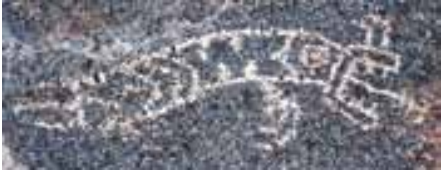

b
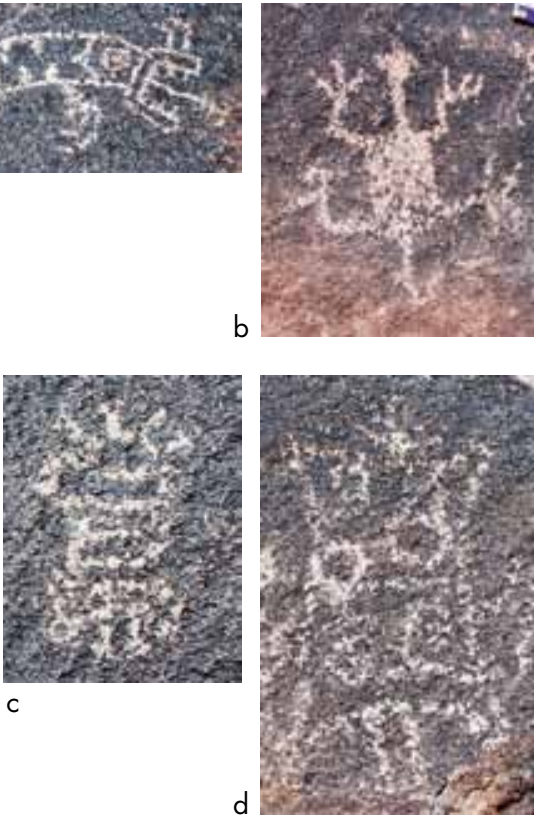

Figura 4. Diseños de filiación trasandina en Los Mellizos (Ciénaga, santamarianos y Aguada): a) "Figura zoomorfa fantástica", soporte 57; b) Reptil Ciénaga, soporte 78; c) Antropomorfo santamariano, soporte 47; d) Antropomorfo santamariano, soporte 146. Figure 4. Designs showing Northwestern Argentinean influence at Los Mellizos (Ciénaga, Santa María and Aguada): a) Imaginary zoomorphic figure, support 57; b) Ciénaga reptile, support 78; c) Santa María buman figure, support 47; d) Santa Maria buman figure, support 146
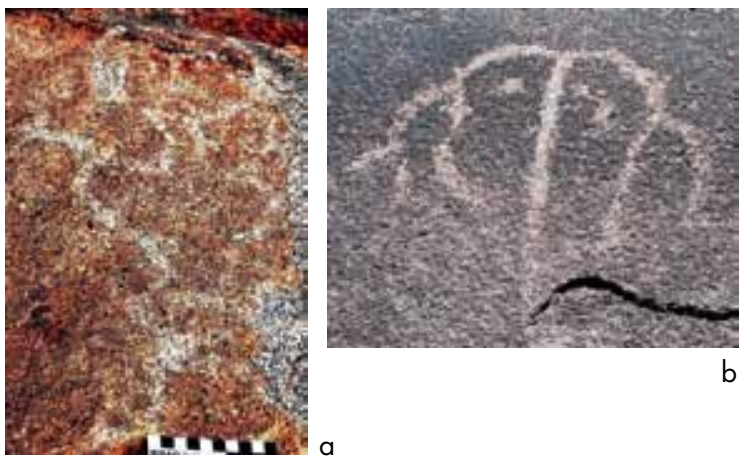

Figura 5. Diseños de filiación Agroalfarero Temprano: a) Máscara ceja continua, soporte 21; b) Máscara ceja continua, soporte 54 Figure 5. Designs showing Early Agro-ceramicist influence: a) Mask of the long eyebrow, support 21; b) Mask of the long eyebrow, support 54.

En tanto, las representaciones zoomorfas se caracterizan por su ejecución mediante principios simétricos y formas geométricas, lo que les da una apariencia estática y rígida. Es evidente la existencia de un patrón bastante consistente en la ejecución de los camélidos. Por ejemplo, el llamado "camélido estilizado", representado mediante la realización de una línea recta horizontal a modo de tronco, con apéndices verticales en traslación horizontal

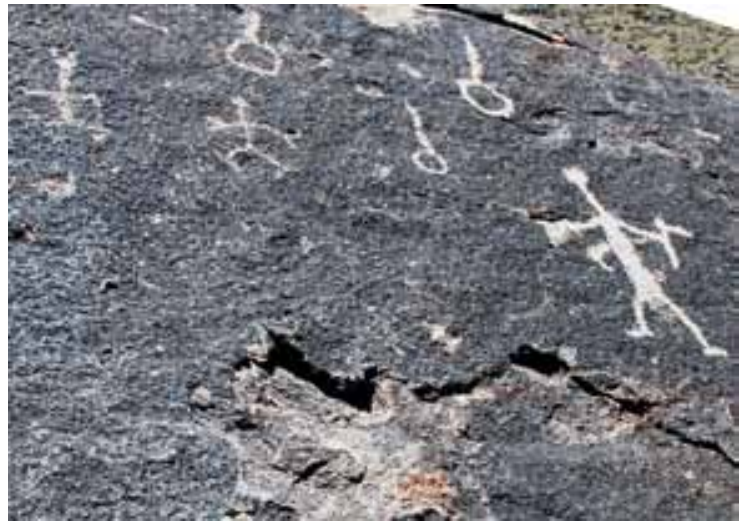

Figura 6. Antropomorfo con hacha en las manos. Sacrificador, soporte 78 .

Figure 6. Human figure with axe in hand, the Sacrificer, support 78 .

a modo de patas (por lo general cuatro), y que presenta apéndices lineales para representar cabeza, hocico, orejas y cola, agrupa al 89,74\% del total de representaciones de camélidos (70 diseños) y un 48,61\% de los diseños figurativos. Otras variantes en la ejecución de camélidos son también de mucho interés, como es el caso del llamado "camélido inka" (fig. 3c) que aunque cuenta con escasa representación (cuatro diseños) se asemeja estrechamente a los camélidos asociados al arte rupestre del Período Inka (ver Sepúlveda 2008).

Otros animales representados en el arte rupestre de Los Mellizos, a los cuales puede asignarse un origen cultural específico consideran la representación de una "figura zoomorfa fantástica" (sensu Gordillo 2009: 110) y el diseño de un reptil, regidos por los principios simétricos de reflexión horizontal y reflexión vertical, respectivamente (figs. $4 \mathrm{a}$ y b). Para ambas representaciones puede sugerirse un origen trasandino. En el caso del reptil, este diseño es muy semejante a representaciones descritas para la Cultura Ciénaga (Sempé 1993) y también Santa María (Fiadone 2006; Nastri 2008, fig. 14), aunque la semejanza iconográfica es mayor con la iconografía de la Cultura Ciénaga. En el ejemplo del arte rupestre del sitio Los Mellizos, la "figura zoomorfa fantástica" (fig. 4a) se caracteriza por reunir caracteres de distintas representaciones zoomorfas, considerando la representación de una entidad mítica con cuerpo de reptil y una fauce felínica, en la cual se distinguen también garras curvas en el sector inferior del cuerpo. Rasgos idénticos fueron descritos por Gordillo (2009: 110) para una representación zoomorfa perteneciente a la iconografía Aguada.

En cuanto a la naturaleza de las relaciones establecidas entre poblaciones trasandinas y poblaciones locales, que explican la presencia de esta iconografía 
en el arte rupestre de Los Mellizos, pensamos que se trata de íconos que poseen una fuerte carga simbólica y que sin duda desempeñaron un importante rol en el ámbito religioso-cultural de estos grupos, lo que explicaría su difusión allende los Andes. Esta circulación de ideas se ve facilitada por la existencia de numerosos pasos naturales que, desde épocas precolombinas hasta el presente, permitieron el traslado de grupos humanos desde y hacia el territorio argentino. Su baja representación apoya la interpretación de que se trata de una circulación de ideas, más que una ocupación efectiva de estos espacios por poblaciones de origen trasandino.

Finalmente, dentro de la categoría de artefactos, destaca la existencia de un cuchillo o tumi, para el que hemos sugerido un origen inkaico (fig. 3b).

\section{Diseños no figurativos}

El estudio de los diseños no figurativos aporta importantes antecedentes para la caracterización del universo representacional del arte rupestre del sitio Los Mellizos. Lo primero que destacamos es la gran representación de esta clase de diseños, que alcanzan a un $77,74 \%$ del total (503 ejemplares), de los cuales un 30,29\% es de naturaleza geométrica simétrica, un $47 \%$ es geométrico no simétrico, un $0,15 \%$ es no geométrico simétrico y un 0,30\% es no geométrico no simétrico.

Respecto a la categoría de diseños geométricos simétricos, observamos que la estructura simétrica con mayor representación es la traslación, que considerada en conjunto (en sus variantes horizontal, vertical y horizontal-vertical) agrupa a un $17,93 \%$ del total de la muestra y a un $59,18 \%$ de los diseños geométricos simétricos. Le sigue en frecuencia de representación la reflexión tipo espejo que, consideradas en conjunto la reflexión vertical y horizontal, alcanzan al 28,57\% de los diseños geométricos simétricos. Debemos precisar que la reflexión vertical supera en un gran margen la representación de la reflexión horizontal (51 diseños frente a cinco diseños, respectivamente). El principio simétrico de la rotación, en tanto, alcanza porcentajes de representación mucho más discretos $(2,16 \%$ del total de diseños) y un 7,14\% de los diseños geométricos simétricos. Finalmente, el empleo conjunto del principio de reflexión vertical y traslación alcanza a un 5,10\% de los diseños geométricos simétricos.

En cuanto a las unidades mínimas empleadas para generar esta categoría de diseños, es interesante notar que existe bastante coincidencia o repetición de ellas, independientemente de la estructura simétrica en que se encuentren insertos, destacándose el empleo de formas geométricas simples que consideran círculos, óvalos, cuadrados, rectángulos, trapecios, y diseños lineales (rectas, curvas, onduladas, zigzag, ángulos), así como combinaciones de estas figuras geométricas y diseños lineales.

Los diseños geométricos no simétricos cuentan con la mayor representación, alcanzando al $47 \%$ del total de diseños analizados (304 ejemplares). Al observar la naturaleza de sus unidades mínimas notamos una fuerte coincidencia con las unidades mínimas utilizadas en los diseños geométricos simétricos, es decir, volvemos a encontrar la presencia de formas geométricas simples (círculos, óvalos, rectángulos, cuadrados, trapecios, triángulos) y diseños lineales (líneas rectas, curvas, onduladas, líneas quebradas, ángulos, cruces). Registran un mayor porcentaje de representación los círculos, los rectángulos y las líneas rectas, curvas y onduladas.

Una categoría interesante la constituyen los "diseños geométricos simétricos complejos", compuesta por 19 variantes resultado de la combinación de diversas unidades mínimas geométricas (13,48\% de los diseños geométricos no simétricos). En estos diseños, en su mayoría, se combinan tres o cuatro formas simétricas diferentes y, excepcionalmente, hasta seis formas geométricas. No obstante, dichas formas mantienen cierta distancia entre sí, encontrándose unidas por líneas, apéndices o intersecciones de las figuras. Una situación diferente presenta la última de estas variantes, que hemos denominado "figuras aglutinadas", que se caracteriza por la representación de diseños abigarrados que combinan varias formas geométricas y diseños lineales, en una suerte de ensamblaje o "mórula". Esta representación alcanza al 5,26\% de los diseños geométricos no simétricos.

La representación de las dos últimas categorías de diseños no figurativos (no geométricos simétricos y no geométricos no simétricos) es muy escasa $(0,15 \%$ y $0,30 \%$, respectivamente), quedando en evidencia las fuertes pautas culturales que rigen este arte visual y que privilegian la representación de figuras geométricas.

Finalmente, hemos dejado para esta última parte la discusión de los antecedentes que nos permiten proponer una asignación cultural a un conjunto de diseños no figurativos pertenecientes al sitio Los Mellizos. Una primera constatación se refiere a que dentro de este conjunto de diseños encontramos mayoritariamente coincidencias formales con la iconografía de grupos culturales del Período Tardío, específicamente de la Cultura Inka, o Diaguita-Inka (ver figs. 3 a-l y tablas 2 a 4). Con las ya mencionadas excepciones de los elementos iconográficos adscribibles a poblaciones prehispánicas trasandinas (culturas Aguada, Ciénaga y Santa María), con porcentajes muy discretos de representación, así 
Tabla 2. Diseños geométricos simétricos figurativos con influencia Inka o Diaguita-Inka: 3.

Table 2. Geometric symmetrical figurative designs with Inka or Diaguita-Inka influence: 3.

\begin{tabular}{|c|c|c|}
\hline Principio simétrico & Diseño & Total \\
\hline \multicolumn{3}{|l|}{ Reflexión vertical } \\
\hline Fitomorfo & Espiga & 1 \\
\hline $\begin{array}{l}\text { Antropomorfo cuerpo } \\
\text { entero }\end{array}$ & $\begin{array}{l}\text { Círculo vacío (cabeza), rectángulo (tronco) y apéndices } \\
\text { rectos (extremidades) }\end{array}$ & 1 \\
\hline $\begin{array}{l}\text { Antropomorfo cuerpo } \\
\text { entero }\end{array}$ & $\begin{array}{c}\text { Cuadrado (cabeza), rectángulo ajedrezado (tronco) y } \\
\text { apéndices rectos (extremidades) }\end{array}$ & 1 \\
\hline
\end{tabular}

Tabla 3. Diseños geométricos simétricos no figurativos con influencia Inka o Diaguita-Inka: 44. Table 3. Geometric symmetrical non-figurative designs with Inka or Diaguita-Inka influence: 44.

\begin{tabular}{|c|c|c|}
\hline Principio simétrico & Diseño & Total \\
\hline \multirow[t]{5}{*}{ Reflexión vertical } & & 7 \\
\hline & Clepsidra & 1 \\
\hline & Cuadrado cruz inscrita & 3 \\
\hline & Círculo con cruz inscrita & 1 \\
\hline & Rectángulo cruz inscrita & 1 \\
\hline Doble reflexión especular & Cuadrados y líneas en zigzag & 1 \\
\hline \multirow[t]{8}{*}{ Traslación horizontal } & & 13 \\
\hline & Círculos vacíos o con punto central & 3 \\
\hline & Rectángulos unidos & 3 \\
\hline & Rectángulos con cruz inscrita & 2 \\
\hline & Líneas onduladas & 1 \\
\hline & Rombos & 1 \\
\hline & Líneas en zigzag & 2 \\
\hline & Equis & 1 \\
\hline \multirow[t]{5}{*}{ Traslación vertical } & & 6 \\
\hline & Círculos con punto central & 1 \\
\hline & Rectángulos vacíos unidos & 1 \\
\hline & Líneas onduladas & 3 \\
\hline & Puntos & 1 \\
\hline \multirow[t]{6}{*}{ Traslación horizontal-vertical } & & 18 \\
\hline & Círculos & 10 \\
\hline & Puntos & 5 \\
\hline & Rectángulos con cuadrado interior & 1 \\
\hline & Rectángulos vacíos unidos & 1 \\
\hline & Cuadrados & 1 \\
\hline Total & & 44 \\
\hline
\end{tabular}

como ciertos diseños adscribibles a poblaciones del PAT ("ceja continua").

Nos detendremos brevemente también en ciertos diseños no figurativos que hemos vinculado con la Cultura Diaguita o Diaguita-Inka, tanto por la naturaleza de las unidades mínimas empleadas en estos diseños, como por los principios simétricos involucrados en su configuración. Pese a su origen claramente Diaguita, no planteamos derechamente su filiación preinkaica debido a que en la Fase Diaguita III o Diaguita-Inka hemos detectado una gran supervivencia de diseños diaguitas preinkaicos, por lo cual una hipótesis que no debemos 
Tabla 4. Diseños geométricos no simétricos no figurativos con influencia Inka o Diaguita-Inka: 13.

Table 4. Geometric asymmetrical non-figurative designs with Inka or Diaguita-Inka influence: 13.

\begin{tabular}{|c|c|}
\hline Diseño & Total \\
\hline Rectángulo vacío de paredes inflectadas & 2 \\
\hline Rectángulo lados rectos & 8 \\
\hline Cuadrado con círculo interior & 1 \\
\hline Cruz doble contorno & 1 \\
\hline Rombo & 1 \\
\hline Total & 13 \\
\hline
\end{tabular}

Tabla 5. Diseños geométricos simétricos no figurativos con influencia Diaguita o Diaguita-Inka: 9.

Table 5. Geometric symmetrical non-figurative designs with Diaguita or Diaguita-Inka influence: 9.

\begin{tabular}{|c|c|c|}
\hline Principio simétrico & Diseño & Total \\
\hline Reflexión vertical & & 2 \\
\hline & Escalonados & 2 \\
\hline Traslación vertical & & 3 \\
\hline & Escalonados & 1 \\
\hline Líneas en zigzag & 2 \\
\hline Reflexión vertical y traslación & Escalonados & 1 \\
\hline Reflexión desplazada y traslación & Escalonados & 2 \\
\hline Reflexión horizontal y traslación & Escalonados & 1 \\
\hline Total & & 9 \\
\hline
\end{tabular}

Tabla 6. Diseños geométricos no simétricos no figurativos con influencia Diaguita o Diaguita-Inka: 1.

Table 6. Geometric asymmetrical non-figurative designs with Diaguita or Diaguita-Inka influence: 1.

\begin{tabular}{|c|c|}
\hline Diseño & Total \\
\hline Laberinto o greca recta & 1 \\
\hline Total & 1 \\
\hline
\end{tabular}

descartar es que estos diseños rupestres se ejecutaran durante la Fase III (1470-1536 DC). Estos diseños incluyen motivos escalonados en reflexión vertical, traslación de escalonados, escalonados en reflexión vertical y traslación, entre otros (tablas 5 y 6 ).

En cuanto a la abundante representación de diseños de origen cusqueño en el sitio de Los Mellizos, consideramos probable que esta actividad forme parte de una estrategia de legitimación del Inka frente a las poblaciones locales, en el proceso de incorporación de estos nuevos territorios al Tawantinsuyu. La profusa representación de esta iconografía foránea da cuenta, mediante un lenguaje visual, no discursivo, de una nueva realidad social en la región que nos ocupa. Asimismo, su expresión espacial, la intervención del paisaje mediante estas marcas icónicas resulta un medio eficaz de hacer presente y reiterar visualmente, a una escala mayor que la manufactura cerámica, el surgimiento de esta nueva realidad social. En efecto, en el arte rupestre de Los Mellizos, tanto de naturaleza figurativa como no figurativa, simétricos y no simétricos, se reconocen un total de 70 diseños asignables a la iconografía Inka o Diaguita-Inka, un 10,82\% del total de diseños registrados en el sitio (ver tablas 2 a 4). Resulta de gran interés esta fuerte presencia de diseños de cronología tardía, considerando además que se recogen no solo las unidades mínimas, sino también sus estructuras simétricas, muchas de ellas presentes en la cerámica Diaguita-Inka.

Hemos de prestar mucha atención en lo sucesivo a la distribución de este arte visual dentro del sitio y a lo largo del valle, dado que nos servirá para acrecentar nuestra comprensión de las interrelaciones y la naturaleza de los contactos establecidos por el Inka con las poblaciones locales, en los que las expresiones de arte rupestre jugaron, al parecer, un importante rol.

\section{CONCLUSIONES PRELIMINARES}

El sitio Los Mellizos es, sin duda, un lugar preponderante en la comprensión de los procesos sociales acaecidos durante la prehistoria del valle del Choapa y, en particular, del río Illapel. Es por ello que una aproximación centrada en el estudio de la lógica de acuerdo a la cual se articula el universo representacional de su arte rupestre es un ejercicio útil y necesario. El análisis de simetría de sus representaciones reveló interesantes coincidencias con patrones decorativos presentes en otras materialidades prehispánicas de datación conocida, que permiten adentrarnos en la asignación cultural de parte de su iconografía y, con ello, esbozar ideas acerca de los procesos de intercambio e interacción cultural. Un resultado interesante se refiere a la alta presencia de diseños adscribibles al Período Diaguita-Inka, que coexisten con otros de naturaleza más local. Esta situación se contrapone a la evidencia registrada en contextos habitacionales del valle de Illapel.

En trabajos anteriores (González 2008) hemos planteado la existencia de estrategias inkas de interacción diferencial en los distintos valles que conforman el territorio diaguita. Ciertamente, en los valles de Elqui y Limarí se aprecia una fuerte aculturación de las poblaciones locales en época Inka, que se ve reflejada en una gran influencia de los patrones decorativos cusqueños, tanto en formas como en decoración cerámica. Por otra 
parte, se percibe una creciente diferenciación social manifestada en la existencia de bienes de prestigio inéditos (aros de plata) y un considerable enriquecimiento de las ofrendas mortuorias. En estas zonas geográficas (Elqui y Limarí), los contextos mortuorios se caracterizan porque los elementos inkas y locales aparecen entremezclados (Cantarutti 2002) y se produce un marcado aumento de la deformación craneana. Todo ello nos sugiere que en estos valles la relación entre el Inka y las poblaciones locales fue privilegiada, generándose una suerte de elite. En tanto, en la frontera sur del territorio diaguita (valle del Choapa), del cual el río Illapel es tributario, la relación establecida por el Inka con los grupos locales se desarrolló de una forma diferente. En el valle de Illapel, las evidencias del Período Inka son bastante más discretas y los asentamientos inkas se segregan de las poblaciones locales. Entonces, la evidencia entregada por el estudio del arte rupestre del sitio Los Mellizos aporta un punto de vista diverso a esta primera constatación, derivada del estudio de sitios habitacionales. Al parecer, en el ámbito propio del arte rupestre (petroglifos) este distanciamiento y segmentación entre lo Inka y los contextos locales ya no resulta tan evidente. Por otra parte, debemos atender también a los mecanismos de difusión de la iconografía cerámica y probablemente textil hacia el soporte rupestre, y cómo este cambio de soporte refleja matices en las estrategias de legitimación del Inka en estos territorios.

\section{NOTAS}

${ }^{1}$ Investigación financiada por Proyecto FONDECYT 1080360.

${ }^{2}$ Respecto al concepto de heterotopía, Troncoso (2001: 14) enfatiza en la definición de Foucault (1986) la característica de tratarse de "un no lugar, un asentamiento que a pesar de tener un emplazamiento definido y en relación con otros asentamientos, se diferencia, convirtiéndose en un lugar absolutamente distinto a todos los otros, generando una ruptura en el espacio y la vida ordinaria, pero que a la vez juega un importante rol en la vida social y organización del espacio cultural de los grupos humanos".

\section{REFERENCIAS}

Artigas, D. \& G. Cabello, 2004. La otra fauna: Los animales olvidados del Choapa. Revista Werken 5: 121-126.

Ballereu, D. \& H. Niemeyer, 1998. Los sitios rupestres de la cuenca alta del río Illapel (Norte Chico, Chile). Chungara 28 (1-2): 319-352.

Cabello, G., 2001. Rostros que hablan: Máscaras del Valle de Chalinga. En Actas del IV Congreso Chileno de Antropología, Tomo II, pp. 1363-1376, Santiago.

Cantarutti, G., 2002. Estadio Fiscal de Ovalle: Redescubrimiento de un sitio Diaguita-Inca en el valle de Limarí (IV Región de Coquimbo, Chile). Memoria para optar al título de Arqueólogo. Departamento de Antropología, Facultad de Ciencias Sociales, Universidad de Chile.
Castillo, G., 1991 Ms. Desarrollo prehispánico en la hoya hidrográfica del río Choapa. Museo Arqueológico de La Serena.

FiAdONE, A., 2006. El diseño indígena argentino. Una aproximación estética a la iconografía precolombina. Buenos Aires: Imprenta La Cuadrícula SRL.

Foucault, M., 1986. Of Other Spaces. Diacritics 16 (1): 22-27.

GALLARDO, F., 2009. Sobre la composición y la disposición en el arte rupestre de Chile. Consideraciones metodológicas e interpretativas. Magallania 37 (1): 85-98.

Gallardo, F.; I. Montt, M. Sepúlveda \& G. Pimentel, 2006. Nuevas perspectivas en el estudio del arte rupestre en Chile. Boletín Sociedad de Investigaciones del Arte Rupestre de Bolivia (SIARB) 20: 77-87.

GonzÁlez, P., 2008. Mediating Opposition: On redefinig Diaguita visual codes and their social role during the Inca Period. En Lenguajes visuales de los Incas, P. González \& T. Bray, Eds., pp. 21-46. BAR International Series 1848, Oxford.

Gordillo, I., 1990. Entre pirámides y jaguares. Ciencia Hoy 8: 18-25, Buenos Aires.

-2009. Dominios y recursos de la imagen. Iconografía cerámica del valle de Ambato. Estudios Atacameños 37: 99-121.

Jackson, D.; D. Artigas \& G. CABello, 2001. Nuevas manifestaciones de petroglifos en la precordillera del Choapa: Técnicas, motivos y significado. En Boletín de la Sociedad Chilena de Arqueología 32: 43-49.

NASTRI, J., 2008. La figura de las largas cejas de la iconografía santamariana. Chamanismo, sacrificio y cosmovisión calchaquí. Boletín del Museo Chileno de Arte Precolombino 13 (1): 9-34.

SAnTos, M., 2005. Arte rupestre, estilo y construcción social del espacio en el noroeste de la península ibérica. Tesis doctoral. Facultad de Geografía e Historia, Universidad de Santiago de Compostela.

SEmpé, M. C., 1993. Principios normativos del estilo de decoración de la cerámica Ciénaga. Publicaciones 20. Instituto de Investigaciones Arqueológicas y Museo, Facultad de Humanidades y Artes, Universidad Nacional de San Juan.

SEPúlvedA, M., 2008. Arte rupestre en tiempos incaicos: Nuevos elementos para una vieja discusión. En Lenguajes visuales de los Incas, P. González \& T. Bray, Eds., pp. 99-111. BAR International Series 1848, Oxford.

TARragó, M.; L. GonZÁlez \& J. NASTRI, 1997. Las interacciones prehispánicas a través del estilo: el caso de la iconografía santamariana. Estudios Atacameños 14: 223-242.

Tilley, C., 1991. Material Culture and Text: The Art of Ambiguity. London: Routledge.

Toro, M., 1996. El arte rupestre en el valle de Illapel. Proyecto Arqueológico Río Illapel, J. Rodríguez, Ed., pp. 116-132. Santiago: Comisión Nacional de Ciencia y Tecnología (CONICYT).

Troncoso, A., 2001. De monumentos y heterotopías: Arte rupestre y paisaje en el curso superior del río Illapel, IV Región, Chile. Boletín del Museo Chileno de Arte Precolombino 8: 9-20.

-2005. Hacia una semiótica del arte rupestre de la cuenca superior del río Aconcagua, Chile Central. Chungara 37 (1): 21-36.

Troncoso, A.; F. Armstrong, F. Vergara, P. Urzúa \& P. Larach, 2008. Arte rupestre en el Valle El Encanto (Ovalle, Región de Coquimbo): Hacia una revaluación del sitio-tipo del Estilo Limarí. Boletín del Museo Chileno de Arte Precolombino 13 (2): 9-36.

Wade, D., 2006. Symmetry. The Ordering Principle. New York: Walker Publishing Company, Inc.

Washburn, D., 1977. A symmetry analysis of Upper Gila Area ceramic design. Papers of Peabody Museum of Archaeology and Etnology, Vol. 68. Cambridge: Cambridge Mass.

- 1983. Toward a theory of structural style in art. En Structure and cognition in art, D. Washburn, Ed., pp. 1-7. Cambridge: Cambridge University Press.

Washburn, D. \& D. CROwE, 1988. Symmetries of culture. Theory and practice of plain pattern analysis. Washington D. C.: University of Washington Press. 
